\title{
Imprints of short distance physics on inflationary cosmology
}

\author{
Richard Easther, ${ }^{1}$ Brian R. Greene, ${ }^{1,2}$ William H. Kinney, ${ }^{1}$ and Gary Shiu ${ }^{3}$ \\ ${ }^{1}$ Institute for Strings, Cosmology and Astroparticle Physics, Columbia University, New York, New York 10027 \\ ${ }^{2}$ Department of Mathematics, Columbia University, New York, New York 10027 \\ ${ }^{3}$ Department of Physics and Astronomy, University of Pennsylvania, Philadelphia, Pennsylvania 19104-6396
}

(Received 15 April 2002; published 26 March 2003)

\begin{abstract}
We analyze the impact of certain modifications to short distance physics on the inflationary perturbation spectrum. For the specific case of power-law inflation, we find distinctive—and possibly observable—effects on the spectrum of density perturbations.
\end{abstract}

DOI: 10.1103/PhysRevD.67.063508

PACS number(s): $98.80 . \mathrm{Cq}$

Inflation stretches quantum fluctuations to astrophysical scales, providing a microscopic mechanism for the formation of galaxies [1]. Most models of inflation yield far more quasi-exponential expansion than the $60 e$-foldings required to solve the difficulties faced by the standard model of the hot big bang. Consequently, astrophysical scales in the present universe map to physical distances in the primordial universe that are exponentially smaller than any conceivable fundamental length. As explained in [2-4], this introduces an implicit assumption into the perturbation spectrum calculation: that spacetime physics and quantum mechanics can be extrapolated to arbitrarily small physical lengths, independently of any fundamental length scale. A fundamental length is predicted by almost all attempted unifications of general relativity with the other fundamental forces of nature, and also by quantum theories of gravity. Naive dimensional arguments identify this scale with the Planck length but, for example, the additional scale introduced by string theory, the string length, can easily be one or two orders of magnitude larger than the Planck length [5], if not more [6]. Thus we ask two important questions: can a fundamental length change the predicted perturbation spectrum and, if so, are these differences detectable observationally? If these questions are answered in the affirmative, they may provide astrophysical tests for theories of nature containing a fundamental length scale, including string theory.

Initial investigations $[3,4]$ relied on assessing the robustness of the usual inflationary spectra to changes in the perturbations' dispersion relations. A different approach was initiated in [7-9] where a nominally string inspired fundamental length appears in the uncertainty relations and provides a short distance cutoff. We showed that the de Sitter space power spectrum predicted for this model is rescaled by a multiplicative constant, observable only if one has independent knowledge of the Hubble constant during inflation [9]. We emphasize that the calculations of [9] and those presented here are not performed within string theory, but instead make use of a standard field theory modified on short scales in a manner inspired by string theory. Our aim is to determine if such short distance scale modifications can yield astrophysically observable signatures.

In almost all non de Sitter inflationary backgrounds the expansion rate is slower than exponential, and the physical horizon size increases with time. We predicted that the spectrum will be changed more dramatically at long wavelengths than at short wavelengths (larger $k$ ), since the impact of the fundamental length increases with its ratio to the physical horizon size [9]. Since the resulting change to the spectrum is more complicated than a simple rescaling, it is - in principle—observable.

This article discusses the perturbation spectrum generated by power law inflation when a fundamental length is inserted into the uncertainty relations. The shape of the spectrum does change, in line with our initial expectation. To this extent, we agree with [8] which presents a qualitative analysis of the same problem. However, the magnitude of the effect drops more slowly as the fundamental length decreases than the analysis of [8] suggests, with important consequences for the possibility of observing this signal.

In broad outline, the procedure for determining the spectrum is not changed by the introduction of a fundamental length. We begin with appropriately normalized field oscillations, which are quantum in origin but obey the classical equations of motion, and extract the spectrum from their asymptotic amplitudes. However, the fundamental length modifies both the evolution equation and the explicit form of the normalization condition.

For wavelengths much greater than the fundamental length, tensor modes $v_{k}$ obey

$$
v_{k}^{\prime \prime}+\left(k^{2}-\frac{a^{\prime \prime}}{a}\right) v_{k}=0,
$$

with $P_{g}^{1 / 2} \propto\left|v_{k} / a\right|$. Scalar modes $u_{k}$ obey a similar equation, with $a$ replaced by $z \equiv a \dot{\phi} / H$. Here $\phi$ is the field responsible for inflation, and $P_{s}^{1 / 2} \propto\left|u_{k} / z\right|$. For the special case of powerlaw inflation, $z \propto a$, so scalar and tensor modes obey identical equations, although their power spectra differ in their normalizations. Since the two types of modes have the same equations of motion at long wavelength, we assume that they also obey the same equations of motion at short wavelength, where the influence of short-distance physics is important, and that any modulations of the power spectrum due to short-distance physics apply identically to tensor and scalar modes. With this assumption, modifications to short distance physics can result in violations of the so-called "consistency condition" for inflation $[10,11]$. While this is reasonable, it is by no means guaranteed: scalar modes are a mix of field and metric fluctuations in an arbitrary gauge [12], where as the tensor modes are purely metric. It is not clear that a short- 
distance cutoff will affect fluctuations of the metric in the same way as fluctuations in an arbitrary scalar field. However, general coordinate invariance implies that we can transform (for example) to a gauge in which even the scalar fluctuations are purely "metric," and if this property is preserved at short distances then the effect of new physics on the scalars and tensors should be identical. Even in the existing literature, metric (gravitational wave) fluctuations can be treated as a generic scalar field at short distances. This is also reasonable, but not inevitable.

Following [7], tensor fluctuations $v_{\tilde{k}}$ obey

$$
v_{\tilde{k}}^{\prime \prime}+\frac{\nu^{\prime}}{\nu} v_{\tilde{k}}^{\prime}+\left(\mu-\frac{a^{\prime \prime}}{a}-\frac{a^{\prime}}{a} \frac{\nu^{\prime}}{\nu}\right) v_{\tilde{k}}=0
$$

where $a$ is the scale factor, the prime denotes differentiation with respect to conformal time $\eta$, while $\widetilde{k}^{i}=a \rho^{i} e^{-\beta \rho^{2} / 2}$ with $\rho^{i}$ being the Fourier transform of the physical coordinates $x^{i}$, and

$$
\mu(\eta, \rho) \equiv \frac{a^{2} \rho^{2}}{\left(1-\beta \rho^{2}\right)^{2}}, \quad \nu(\eta, \rho) \equiv \frac{e^{3 \beta \rho^{2} / 2}}{\left(1-\beta \rho^{2}\right)} .
$$

When evaluating the derivatives of $v_{\tilde{k}}$ with respect to $\eta$, we are holding $\tilde{k}$ (and not the usual comoving momentum $k$ ) fixed with time. It is therefore convenient to express $\mu$ and $\nu$ in terms of $\tilde{k}$ by introducing the Lambert $W$ function [14], which is defined so that $W\left(x e^{x}\right)=x$ :

$$
\mu=-\frac{a^{2}}{\beta} \frac{W(\zeta)}{[1+W(\zeta)]^{2}}, \frac{\nu^{\prime}}{\nu}=\frac{a^{\prime}}{a} \frac{W(\zeta)[5+3 W(z)]}{[1+W(\zeta)]^{2}},
$$

where $\zeta=-\beta \tilde{k}^{2} / a^{2}$.

For power law inflation

$$
a(t)=t^{p}, \quad a(\eta)=\left(\frac{\eta}{\eta_{0}}\right)^{q}, \quad q=\frac{p}{1-p}
$$

For inflation to occur, we need $p>1$.

The cutoff is introduced by requiring that $\rho^{2} \leqslant 1 / \beta$, motivated by the notions of a minimum distance in string theory and the so-called "stringy uncertainty principle" [15]. Fluctuations with comoving wave number $k$ reach the cutoff $\rho^{2}$ $=1 / \beta$ at $\eta_{k}$, where

$$
\eta_{k}=\eta_{0}\left(e \beta \tilde{\kappa}^{2}\right)^{1 / 2 q}=\frac{1}{1-p}\left(e \beta \tilde{\kappa}^{2}\right)^{1 / 2 q}
$$

where the implicit definition of $\eta_{0}$ comes from setting $a(t)$ $=1$ when $t=1$, and $t$ is the usual physical time.

Writing $\eta=\eta_{k}(1-y)$ in order to extract the $k$ dependence and abbreviating $W(\zeta)$ as $W$, we have

$$
\begin{aligned}
\ddot{v}_{\tilde{k}}- & \frac{q}{1-y} \frac{W(5+3 W)}{(1+W)^{2}} \dot{v}_{\tilde{k}}-\left(\frac{(1-y)^{2 q} \eta_{k}^{2 q+2} W}{\beta \eta_{0}^{2 q}(1+W)^{2}}+\frac{q(q-1)}{(1-y)^{2}}\right. \\
& \left.+\frac{q^{2}}{(1-y)^{2}} \frac{W(5+3 W)}{(1+W)^{2}}\right) v_{\tilde{k}}=0 .
\end{aligned}
$$

In the de Sitter case, $k$ can be eliminated from the equation of motion, but this cannot be done here. During power-law inflation, different modes sample a different value of the Hubble constant as they cross the horizon, and this will be reflected in the scale-dependent modifications to the spectrum we will observe. Despite this, our analysis of the de Sitter case [9] can easily be adapted to the power-law problem.

When $\zeta=-1 / e, W(\zeta)$ has a branch point [14]. Physically, this is the moment when $y=0\left(\eta=\eta_{k}\right)$ and the fluctuation with wavelength $k$ is "created." As in [9], we solve for the leading behavior of $v_{k}$ by extracting the most singular terms of the equation of motion,

$$
\ddot{v}_{\tilde{k}}-\frac{1}{2 y} \dot{v}_{\tilde{k}}+\frac{A_{k}}{y} v_{\tilde{k}}=0,
$$

where overdots denote derivatives with respect to $y$, and

$$
A_{k}=-\frac{1}{q} \frac{\eta_{k}^{2 q+2}}{4 \beta \eta_{0}^{2 q}}-\frac{q}{2}
$$

The solution to Eq. (8) is

$$
v_{\tilde{k}}^{(0)}(y)=y^{3 / 4}\left(C H_{-3 / 2}^{(2)}\left(2 \sqrt{A_{k} y}\right)+D H_{-3 / 2}^{(1)}\left(2 \sqrt{A_{k} y}\right)\right) .
$$

Here, $H_{-3 / 2}^{(2)}$ is the second Hankel function; $C$ and $D$ are constants. The first Hankel function is its complex conjugate $H_{q / 2-1}^{(1)}=H_{q / 2-1}^{(2) *}$.

The solution is normalized by the Wronskian condition

$$
v_{\tilde{k}}(\eta) v_{\tilde{k}}^{*^{\prime}}(\eta)-v_{\tilde{k}}^{*}(\eta) v_{\tilde{k}}^{\prime}(\eta)=i\left(1-\beta \rho^{2}\right) e^{-3 \beta \rho^{2} / 2}
$$

Using Hankel function identities [16] we deduce

$$
|C|^{2}-|D|^{2}=-\eta_{k} \pi \sqrt{-q} e^{-3 / 2} .
$$

This, together with Eqs. (10) and (13), gives the general result for all possible boundary conditions. More specifically, though, we must fix $C$ and $D$, i.e. we must specify boundary conditions for Eq. (8) or, equivalently, the form of the vacuum state. Ultimately, a more complete understanding of short scale physics would allow a first principles selection of boundary conditions. Here we simply note that if $D \neq 0$, the spectrum never approaches the exact power-law form, even when the Hubble parameter is arbitrarily small. Consequently, $D=0$ is the only vacuum choice with constant coefficients that reduces to the Bunch-Davies vacuum at low energies. We thus focus our analysis on the "minimal" choice that $D=0$ for all $k$, but we stress that this choice is 


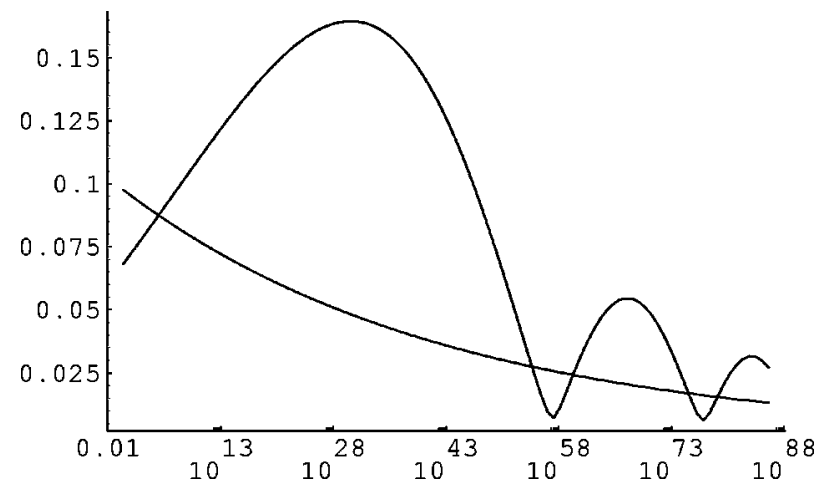

FIG. 1. The scalar spectrum, $P_{s}^{1 / 2}(k)$ is plotted against $k$, with $\sqrt{\beta}=100$ and $p=100$, where $k=1$ corresponds to $k_{\text {crit }}$. The standard power law spectrum is plotted for comparison (smooth line). It should be noted that for any reasonable parameter values, these large modulations correspond to physical scales far bigger than the present horizon volume, and will have no observable impact on present-day cosmology.

not unique: $C$ and $D$ could depend on $\beta, k$ or $H$ in a such a way that the constraint of Eq. (12) was always satisfied, and that $D$ was non-zero at high energies, and vanishing sufficiently rapidly as $\beta \rightarrow 0$ to avoid any experimental constraints on non-zero $D$. Our results obviously depend on this choice and this issue certainly deserves further investigation. Finally, we point out that setting $q=-1$ in Eq. (12) reproduces the de Sitter result, after reconciling the normalization factors.

We solve the mode equations numerically $[9,13]$ and match the numerical solution to the approximate analytical form, including sub-leading corrections, near $y=0$. We obtain the scalar spectrum by solving the mode equations for multiple values of $k$, and then extracting the necessary late time limit to compute

$$
P_{g}^{1 / 2}=\sqrt{\frac{k^{3}}{2 \pi^{2}}}\left|\frac{v_{k}}{a}\right|_{k=a H}, \quad P_{s}^{1 / 2}=\sqrt{32 \pi p} P_{g}^{1 / 2}
$$

where we have obtained the scalar spectrum from the tensor one, as outlined above.

The spectrum is only well defined if the minimum length $(\sqrt{\beta})$ is less than the horizon size $(1 / H)$, or $\sqrt{\beta} H$ is less than unity. The critical mode, $\kappa_{\text {crit }}$, that crosses the horizon at the moment when $\sqrt{\beta} H=1$ is

$$
k_{\text {crit }}=p\left(e \beta p^{2}\right)^{(p-1) / 2} .
$$

For large values of $p, \kappa_{\text {crit }}$ is enormous. This reflects the massive amount of inflation that takes place between the Planck time ( $t=1$ in natural units) and the moment at which $\sqrt{\beta} H=1$; the numerical value of $k$ can always be rescaled by redefining $a_{0}$, the value of $a$ when $t=1$.

Figure 1 shows the spectrum for the longest modes, with $p=100$. There is a large modulation in the spectrum, corresponding to the slow decrease in $H$ as the universe evolves. However, these modes have a much larger amplitude than those contributing to the cosmic microwave background
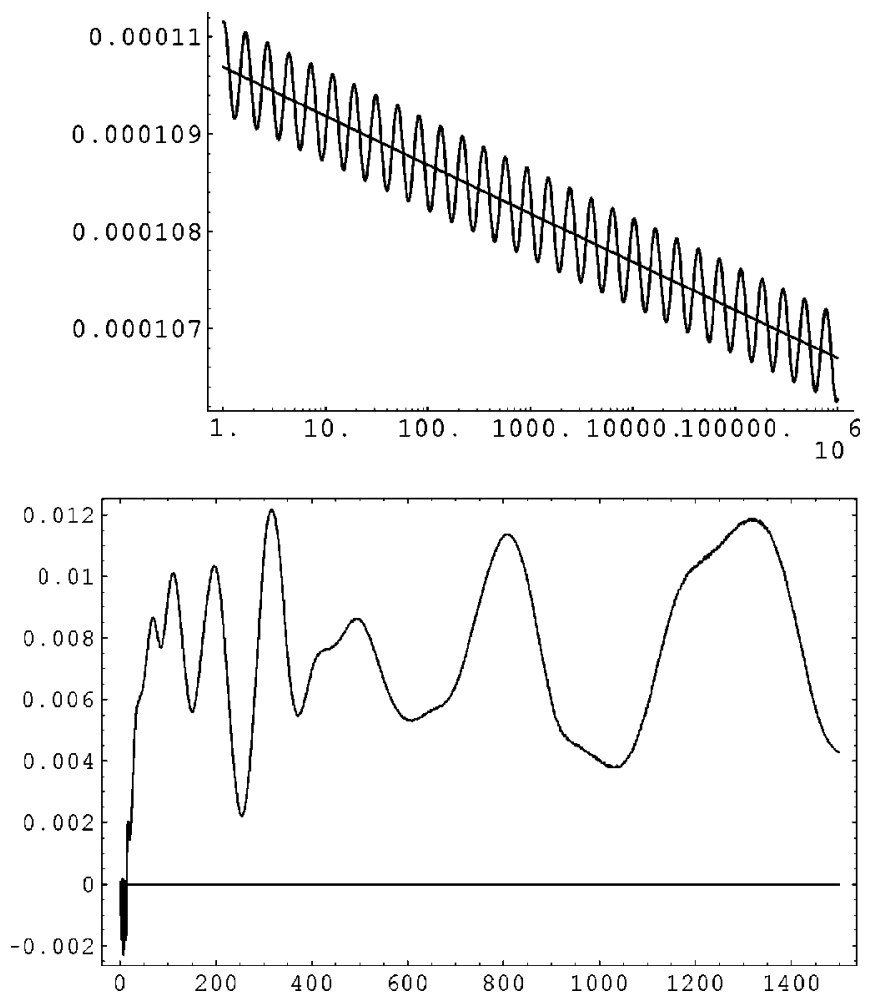

FIG. 2. The top plot plots $P_{s}^{1 / 2}(k)$ against $k$ for $\sqrt{\beta}=100$ and $p=500$, and with the straight line showing the standard power-law result. The modulation of the spectrum has an amplitude of $0.4 \%$ of the overall signal. The bottom plot shows the percentage change in the $C_{l}$ values (plotted against $l$ ) computed from this spectrum, relative to the spectrum calculated in the absence of a fundamental length.

(CMB) power spectrum and structure formation. Figure 2 displays the spectrum with $p=500$ and a "window" of $k$ values with amplitudes of the same order as the modes which are the precursors to structure formation. We have not carefully normalized this spectrum (which requires assumptions about the dark matter composition, $\Omega_{\Lambda}$, etc.), since any signal of trans-Planckian physics is much smaller than the uncertainty in currently available data. Instead we assume that $10^{-6} \lesssim \Delta T / T \lesssim 10^{-5}$, which-given that $(\Delta T / T)^{2} \simeq P_{s} / 180$ [17] — corresponds to $1.5 \times 10^{-5} \lesssim P_{s}^{1 / 2} \lesssim 1.4 \times 10^{-4}$.

The standard power-law spectrum is modulated by an "oscillation" whose amplitude and wavelength depend on both the fundamental length $\sqrt{\beta}$ and the power-law parameter, $p$. The oscillations are attributable to successive modes undergoing increasing numbers of periods between the initial time and horizon exit, with a full extra period corresponding to a single oscillation in the spectrum.

The amplitude and period (in $\log k$ ) of the oscillations are roughly proportional to $\sqrt{\beta}$. In principle $\sqrt{\beta}$ is predicted by fundamental theory, but from our perspective here it is a free parameter. If $\sqrt{\beta}$ is identified with the string scale, it could conceivably be two orders of magnitude longer than the Planck length, and we use this value in the numerical plots. Observationally, the key parameter is the ratio of the fundamental length to the Hubble radius $\sqrt{\beta} H$. In power-law inflation (and any other non de Sitter model) $H$ is a slowly 
changing parameter. The observationally relevant range of $H$ is fixed by the amplitude of the power spectrum, which is deduced from observations of the CMB and large scale structure.

The rate of change of $H$ is determined by $p$, and as $p$ increases the wavelength of the fluctuations in the spectrum increases while their amplitude goes down. This accords with our physical understanding of the oscillations: for a fixed value of $H$ (and thus $P_{s}^{1 / 2}$ ), $\dot{H}$ decreases with increasing $p$. Thus the wavelength of the oscillations (in $\log k$ ) increases with $p$, since $H$ at horizon exit changes more slowly with $k$ at larger $p$. The variation in the amplitude arises because we are effectively holding $H$ fixed at horizon exit, but $H\left(\tau_{k}\right)$ decreases as $p$ is increased. Consequently, the effective value of $\beta H^{2}$ for a given mode decreases as $p$ is increased, which accounts for the $p$ dependence of the amplitude of the oscillations. The oscillations do not vanish as $p$ becomes arbitrarily large, although their wavelength becomes arbitrarily long, and we approach the de Sitter limit where the spectrum is shifted by a constant multiplicative factor.

In Fig. 2, $p=500$ the spectrum is almost flat, and the $C_{l}$ values that would be measured by CMB experiments are modified by between $0.5 \%$ and $1 \%$. A signal of this size lies at the limits of detectability, even with ideal experiments, and would be swamped by cosmic variance at all but the largest values of $l$. Existing constraints on the spectral index put a weak lower bound on $p$ of around 20. With this value, the oscillations' wavelength is so short that the resulting spectrum appears to include a random noise term when plotted over the range of $k$ values relevant to structure formation.

Despite the extreme challenge and perhaps near impossibility of detecting an effect of this size for reasonable values of $\beta$, the conclusions of this article are still much more optimistic than we might have otherwise expected. First, and in accord with our previous de Sitter calculation, we find that the magnitude of the modification to the spectrum is a function of $\left(\beta H^{2}\right)^{n}$, where $n$ appears to be slightly smaller than $1 / 2$. This disagrees with [8], in which it is argued that $n$ is roughly unity. However, [8] relies on a WKB approximation to the mode equation and chooses the vacuum to be the purely "- " WKB solution. We have decomposed our numerical solutions into the two WKB solutions at a time when the WKB approximation holds well, and the actual solution (using the initial conditions described above and also advocated by [8]) contains a mixture of both WKB solutions, where the coefficient on the "+" solution scales like $\left(\beta H^{2}\right)^{n}$ with $n \leq 0.5$. It is this mode mixing of the standard WKB solutions, with a mixing coefficient of order $\sqrt{\beta} H$, that accounts for the magnitude of our results. Thus the pure "- " WKB approximation is not consistent with these initial conditions, perhaps explaining why the estimate of [8] for the impact of the fundamental length on the spectrum is significantly less than we find here.

We have assumed that the minimum length lies somewhat below the Planck scale. While this is justifiable from a stringy perspective, if we put the fundamental length equal to the Planck length $(\sqrt{\beta}=1)$ the effect we see decreases significantly. Moreover, as mentioned, the equations we solve are not derived from string theory and hence there is no guarantee that they are the ones that quantum gravity will give us. Nevertheless, we find it encouraging that whereas there are 16 orders of magnitude separating the Planck scale from conventional accelerator experiments, the string scale modifications we study here yield cosmological effects that may be only one or two orders of magnitude below the threshold of observability.

In principle existing $\mathrm{CMB}$ measurements put experimental restrictions on a portion of the $(\beta, p)$ plane. Given the accuracy of current data, the constraints on $\beta$ would be extremely weak, and we have not performed this calculation. As CMB data and surveys of large scale structure (and our ability to work backward from the observed to the primordial spectrum) improve, it may become possible to place meaningful restrictions on short scale physics using astrophysical and cosmological data.

It is natural to ask whether we can do a full string theoretic version of this calculation. One approach would be to study the full two-point functions of graviton and inflation string excitations, either from string field theory or along the lines of Ref. [18]. These quantities are sensitive to the large number of high energy degrees of freedom found in string theory, another inherently stringy feature.

We thank Robert Brandenberger and Jens Niemeyer for discussions. The work of B.G. is supported in part by DOE grant DE-FG02-92ER40699B and the work of G.S. was supported in part by the DOE grants DE-FG02-95ER40893 and DE-EY-76-02-3071 and the University of Pennsylvania School of Arts and Sciences Dean's funds. ISCAP gratefully acknowledges the generous support of the Ohrstrom Foundation.
[1] V.F. Mukhanov and G.V. Chibisov, JETP Lett. 33, 532 (1981); Sov. Phys. JETP 56, 258 (1982); S.W. Hawking, Phys. Lett. 115B, 295 (1982); A.A. Starobinsky, ibid. 117B, 175 (1982); A.H. Guth and S.-Y. Pi, Phys. Rev. Lett. 49, 1110 (1982); J. Bardeen, P.J. Steinhardt, and M. Turner, Phys. Rev. D 28, 679 (1983); V.F. Mukhanov, JETP Lett. 41, 493 (1985).

[2] R. Brandenberger, hep-ph/9910410.

[3] J. Martin and R.H. Brandenberger, Phys. Rev. D 63, 123501 (2001); R.H. Brandenberger and J. Martin, Mod. Phys. Lett. A 16, 999 (2001).
[4] J.C. Niemeyer, Phys. Rev. D 63, 123502 (2001); J.C. Niemeyer and R. Parentani, ibid. 64, 101301 (2001).

[5] V.S. Kaplunovsky, Nucl. Phys. B307, 145 (1988); B382, 436(E) (1992).

[6] N. Arkani-Hamed, S. Dimopoulos, and G. Dvali, Phys. Lett. B 429, 263 (1998); I. Antoniadis, N. Arkani-Hamed, S. Dimopoulos, and G. Dvali, ibid. 436, 257 (1998); G. Shiu and S.-H.H. Tye, Phys. Rev. D 58, 106007 (1998).

[7] A. Kempf, Phys. Rev. D 63, 083514 (2001).

[8] A. Kempf and J.C. Niemeyer, Phys. Rev. D 64, 103501 (2001). 
[9] R. Easther, B.R. Greene, W.H. Kinney, and G. Shiu, Phys. Rev. D 64, 103502 (2001).

[10] L. Hui and W.H. Kinney, Phys. Rev. D 65, 103507 (2002).

[11] G. Shiu and S.H. Tye, Phys. Lett. B 516, 421 (2001).

[12] V.F. Mukhanov, H.A. Feldman, and R.H. Brandenberger, Phys. Rep. 215, 203 (1992).

[13] J. Adams, B. Cresswell, and R. Easther, Phys. Rev. D 4, 123514 (2001).

[14] R. Corless, G. Gonnet, D. Hare, D. Jeffrey, and D. Knuth, Adv. Comput. Math. 5, 329 (1996).

[15] G. Veneziano, Europhys. Lett. 2, 199 (1986); D. Gross and P.
Mende, Nucl. Phys. B303, 407 (1988); D. Amati, M. Ciafaloni, and G. Veneziano, Phys. Lett. B 216, 41 (1989); R. Guida, K. Konishi, and P. Provero, Mod. Phys. Lett. A 6, 1487 (1991).

[16] Handbook of Mathematical Functions with Formulas, Graphs and Mathematical Tables, edited by M. Abramowitz and I.A. Stegun (Dover, New York, 1965).

[17] M.S. Turner, M. White, and J.E. Lidsey, Phys. Rev. D 48, 4613 (1993).

[18] A.G. Cohen, G.W. Moore, P. Nelson, and J. Polchinski, Nucl. Phys. B267, 143 (1986). 